\title{
Neutrophils in Gliomas
}

\author{
Matteo Massara',2, Pasquale Persico ${ }^{1}$, Ornella Bonavita ${ }^{1,2}$, Valeria Mollica Poeta ${ }^{1,3}$, \\ Massimo Locati ${ }^{1,2}$, Matteo Simonelli, ${ }^{1,3}$ and Raffaella Bonecchi',3* \\ ${ }^{1}$ Humanitas Clinical and Research Center, Rozzano, Italy, ${ }^{2}$ Department of Medical Biotechnologies and Translational \\ Medicine, Università degli Studi di Milano, Milan, Italy, ${ }^{3}$ Humanitas University, Rozzano, Italy
}

Neutrophils are the most abundant white blood cells and are the first recruited to inflammatory sites. Neutrophils are an important component of the tumor stroma and can exert both anti-tumoral and pro-tumoral activities, depending on their maturation and activation state. In human gliomas, the number of circulating and infiltrating neutrophils correlates with the severity of the disease, indicating a prognostic and possible pro-tumoral role for these leukocytes. In glioma preclinical models, neutrophils promote tumor growth and orchestrate the resistance to anti-angiogenic therapies. Nevertheless, recent data indicate that neutrophils can be activated to directly kill tumor cells or to orchestrate the anti-tumoral response. Here, we review current knowledge about the

OPEN ACCESS

Edited by:

Ji Ming Wang,

National Cancer Institute at Frederick, United States

Reviewed by:

Paul Proost,

KU Leuven, Belgium

Remo Castro Russo,

Universidade Federal de Minas

Gerais, Brazil

*Correspondence:

Raffaella Bonecchi

raffaella.bonecchi@

humanitasresearch.it

Specialty section:

This article was submitted

to Cytokines and Soluble

Mediators in Immunity,

a section of the journal

Frontiers in Immunology

Received: 11 July 2017

Accepted: 03 October 2017

Published: 26 October 2017

Citation:

Massara M, Persico P, Bonavita O, Mollica Poeta V,

Locati $M$, Simonelli $M$ and Bonecchi R (2017)

Neutrophils in Gliomas.

Front. Immunol. 8:1349. doi: 10.3389/fimmu.2017.01349 role of neutrophils in glioma and their possible involvement in new strategies to improve current cancer therapies.

Keywords: neutrophils, glioma, inflammation and cancer, immunotherapy, cancer immunotherapy, chemokines

\section{INTRODUCTION}

Gliomas represent approximately $80 \%$ of malignant brain tumors with an estimated annual incidence of 6.6 per 100,000 individuals in the USA. Before publication of the revised World Health Organization (WHO) Classification of Tumors of the central nervous system (CNS) in 2016, gliomas were historically classified into four major histological groups (grades I-IV), according to their microscopic characteristics (such as cytological atypia, anaplasia, mitotic activity, microvascular proliferation, and necrosis) and clinical behavior. These tumors were also divided into astrocytomas (WHO grade I-IV), oligodendrogliomas (WHO grade II-III), and mixed oligoastrocytomas (WHO grade II-III) depending on their putative cells of origin.

Grade IV glioma or glioblastoma (GBM) is the most common and lethal primary brain tumor in adults and notoriously has a highly aggressive clinical course with a median survival time ranging from 12.2 to 18.2 months and less than $5 \%$ of patients alive after 5 -year from initial diagnosis. In contrast, gliomas of lower grade (WHO grade II-III), accounting for approximately one-third of all gliomas, are usually less aggressive tumors with a highly variable clinical course that is not adequately predicted on the basis of their histologic class. Some are indolent tumors, but others quickly progress to a more aggressive form or to GBM over years. Genome-wide molecular-profiling studies have now revealed a comprehensive mutational landscape for all major types of human gliomas occurring in adults and in children (1). In the revised 2016 WHO Classification of Tumors of the CNS, the advances in our molecular understanding of gliomas are integrated in a novel, multilayered approach to disease categorization that incorporates both histological and molecular information to better define many tumor entities and their prognosis.

Despite all these molecular efforts, the current standard of care for newly diagnosed GBM has remained unchanged over the past 10 years and includes maximal safe resection followed by radiotherapy plus concomitant and adjuvant chemotherapy with temozolomide (2). Current feasible therapeutic options for lower-grade gliomas vary with the extent of resection, histologic class, grade, and the results of molecular testing (IDH mutations and $1 \mathrm{p}$ and $19 \mathrm{q}$ co-deletion) 
and include clinical monitoring, radiation alone, sequential, or concomitant chemo-radiation. Outcome for patients with recurrent/progressive disease is dismal, and no therapeutic intervention has been associated with durable survival benefit. According to that, considerable interest has been directed in the development of more effective therapies including innovative therapeutic approaches.

However, clinical trials evaluating intensifying cytotoxic therapy, targeting dysregulated cell signaling pathways (3), and blocking angiogenesis (4) failed to prolong patients' survival. Giving the exciting and durable clinical benefits achieved for a number of other challenging cancers, recent efforts have been directed toward the modulation of the immune system by various therapeutic interventions also in the neuro-oncology field. Until now immunotherapeutic strategies for gliomas were only partially successful, further underlying the strong immunosuppressive potential of these tumors (5). This article aims to review the role of neutrophils in glioma, their role in the immunosuppression, and resistance to therapies and possible strategies to harness their anti-tumoral potential to fight gliomas.

\section{THE IMMUNE RESPONSE IN THE CNS}

The CNS has traditionally been recognized as an immuneprivileged site lacking the potential for immunosurveillance due to the presence of the blood-brain barrier (BBB) and the absence of a conventional lymphatic system. Lack of immune response is provided by preclinical data demonstrating the prolonged survival of tissues grafted into brain that were otherwise rejected rapidly by the immune system when grown outside the CNS. However, this paradigm has been recently challenged and it is becoming increasingly clear that the CNS interacts dynamically with the systemic immune system. Recent data indicate that leukocytes can traffic to the CNS, even in the presence of an intact $\mathrm{BBB}$, and that $\mathrm{CNS}$ antigens can reach peripheral lymph nodes by draining cerebrospinal fluid (CSF) through VirchowRobin spaces $(6,7)$. Furthermore, in 2015, the presence of lymphatic vessels within the dural sinuses capable of shuttling leukocytes between CSF and deep cervical lymphatic vessels has been reported (8).

In homeostatic conditions, leukocytes are only present in the CSF and in the vessels and they do not enter in the brain parenchima. The only immune cells that are present in the CNS are parenchymal macrophages (microglia) that have a prenatal origin, are long-lived, and have no turnover with blood monocytes (9). Furthermore, in the brain, there are nonparenchymal macrophages named perivascular, meningeal, and choroid plexus macrophages that are strategically located at the CNS barrier and are thought to modulate leukocyte traffic and to sense blood danger signals (9).

Naive T cells do not readily enter the normal or inflamed CNS. Rather, T cells need to be highly activated to enter the brain, suggesting that they need to be primed in the periphery to be able to enter to this site (10). Inflammatory monocytes infiltrate the brain during autoimmune and neurodegenerative diseases with an intact $\mathrm{BBB}$, indicating that they are actively recruited to the CNS. The CCR2/CCL2 axis seems to be crucial for the trafficking of monocytes across the BBB (11). Neutrophils can infiltrate the brain with a LFA-1-dependent mechanism during Alzheimer disease (12), and in a murine model of sepsis, neutrophils are actively recruited to the brain by the chemokines, such as CXCL1, CXCL2, and CXCL3 (13). In has to be noted that in humans the chemokines involved in brain recruitment of neutrophils can be different because mice do not express CXCL8 the major human neutrophil attractant.

\section{THE ROLE OF THE IMMUNE SYSTEM IN GLIOMA}

By interfering with different aspects of tumor growth and responsiveness to therapy, the interplay between tumor cells and the immune system is emerging as a key modulator of tumor biology and one of the major determinants of cancer pathogenesis and progression (14). Increasing evidence indicates that tumors develop and progress by co-opting seemingly normal host cell types that reside in or are recruited to tumor microenvironment.

In glioma patients, there is a profound and generalized immunosuppression, particularly within the context of cell-mediated immunity (Figure 1). The systemic immunosuppression is driven by overexpression by the glioma cells of soluble factors, such as prostaglandin E2, TGF- $\beta$, indoleamine 2,3-dioxygenase, and IL-10, all of which lead to decrease T-cell responsiveness to pro-inflammatory signals and ineffective presentation of tumor antigens by antigen-presenting cells (APCs). On the other side, the same mediators drive the recruitment of immunosuppressive cells, such as regulatory $\mathrm{T}$ cells and myeloid suppressor cells, to the tumor microenvironment. Moreover, corticosteroids (often used in glioma patients to diminish cerebral edema) are immunosuppressive, and temozolomide chemotherapy and radiotherapy can trigger prolonged lymphopenia. Several immunosuppressive alterations do occur within the glioma microenvironment. Glioma cells can down-regulate their own MHC-I complexes, thus becoming "invisible" to the adaptive immune system, as well as increase expression of immune checkpoint regulators, such as PD-1 (15-17). Specific blockade of PD-1 in combination with radiotherapy induced tumor regression and promoted long-term survival in animal models of glioma (18). The combination therapy of anti-CTLA-4 and anti-PD-1 has resulted in a $75 \%$ long-term remission in an orthotopic, immunocompetent murine GBM model. In this model, there were increased numbers of activated $\mathrm{CD}^{+}$and $\mathrm{NK}$ cells with reductions in suppressive immune cells in the tumor microenvironment (19). Additionally, combined blockade of CTLA-4, PD- 1 and indoleamine dioxygenase, and anti-CTLA- 4 therapy alone have resulted in long-term survival in other murine models of GBM and combined blockade of CTLA-4 and IL-12 was shown to increase numbers of effector T cells and decrease Tregs (20-22).

Multiple strategies to overcome immunosuppression and boost anti-tumor immune responses are now under evaluation in clinical trials, including vaccination with tumor-specific or tumor-associated peptides (or with administration of autologous 


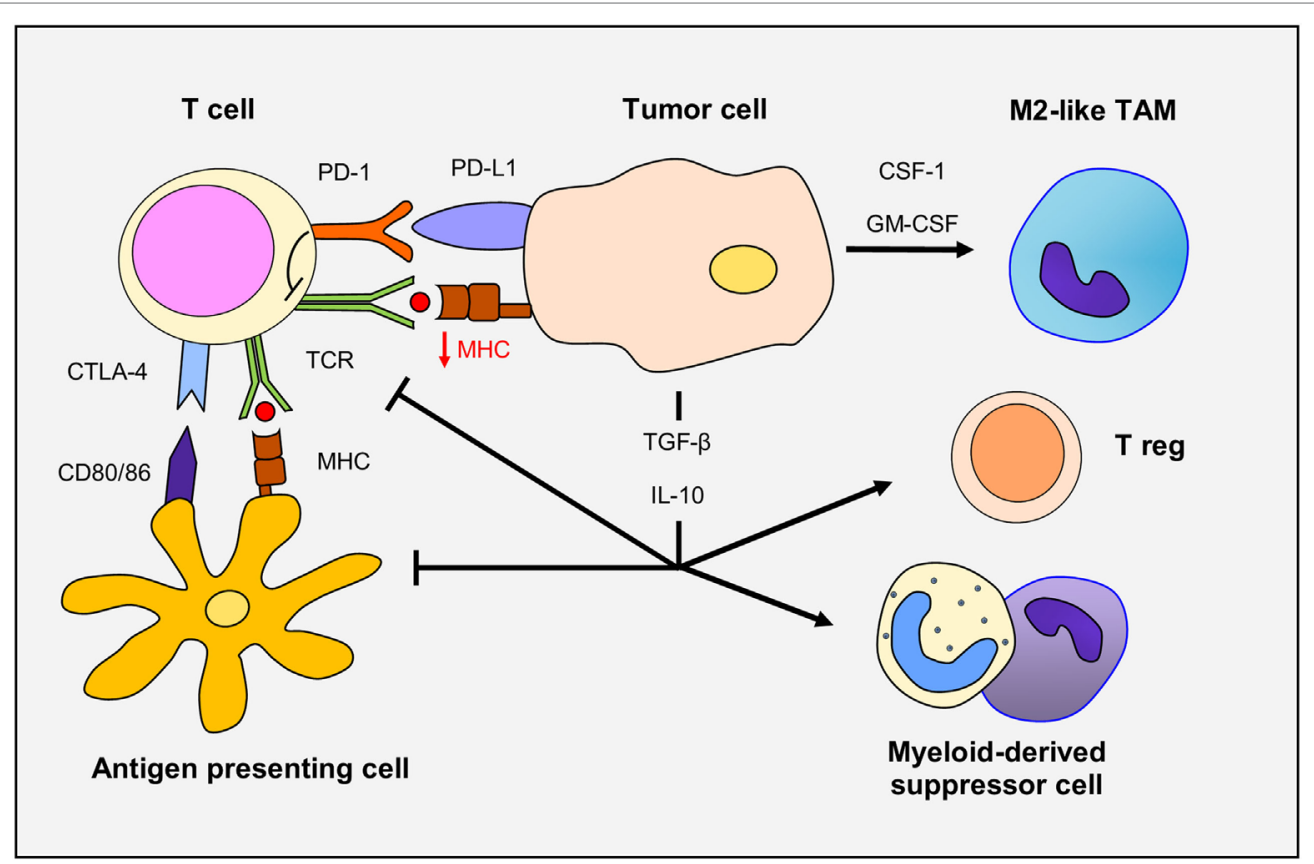

FIGURE 1 | The immune response in gliomas. Glioma cells overexpress TGF- $\beta$ and IL-10, immunosuppressive cytokines that decrease T-cell responsiveness and antigen-presenting cell function. Glioma cells down-regulate $\mathrm{MHC}$-I and upregulate programmed death ligand-1 (PD-L1) expression leading to defective T-cell responsiveness. TCR activity is also dampened through CTLA-4 expression. TGF- $\beta$ and IL-10 promote the recruitment and differentiation of regulatory T cells. Finally, secretion of CSF-1 and GM-CSF by glioma cells increase macrophage recruitment and polarization to M2-like phenotype.

APCs pulsed with such peptides), immunomodulatory monoclonal antibodies that target T-cell inhibitory signaling pathways to enhance physiological anti-tumor responses and adoptive T-cell transfer, which involves ex vivo clonal expansion or genetic engineering of T cells (23).

In addition to strategies aimed to T-cell activation, efforts were also done to target tumor-associated macrophages to inhibit glioma progression. Indeed, macrophages appear to be the main infiltrating immune cells in gliomas. Several approaches have been performed using inhibitors of the CSF-1 receptor (CD115). This treatment blocks maturation of monocytes and polarization of macrophages toward an M2-like pro-tumoral phenotype and resulted in increased survival of the GBM-bearing mice $(24,25)$. Other myeloid cells are present in the glioma microenvironment, including neutrophils (26). These leukocytes, until a few years ago, were considered as innocent bystanders in the tumor stroma, and their role in the tumor context was not investigated. On the contrary, emerging evidence indicates that neutrophils can play an important role in tumors having both pro-tumoral and anti-tumoral functions.

\section{NEUTROPHILS AND CANCER}

Neutrophils are the most abundant leukocyte population in the bloodstream, and they are essential effectors of the inflammatory response and defense against pathogens. Even if neutrophils are mostly considered for their anti-microbial functions, it is now evident that they exert a broader role in the immune response (27).
In the cancer setting, neutrophils were considered for a long time only for their pro-tumoral activities because in many cancer types, neutrophilia and an elevated ratio of peripheral neutrophils-to-lymphocytes counts (NLR) are associated with poor prognosis $(28,29)$. They were considered the main cell type mediating immunosuppression and tumor promotion. Only recently their anti-tumoral potential has been reconsidered for several reasons. First, clinical studies have indicated that neutrophil infiltration is associated with a favorable prognosis in gastric cancer (30), in colorectal cancer (31), and in early stages of non-small-cell lung carcinoma (32). Second, preclinical data have indicated that circulating and tumor-infiltrating neutrophils are not a homogeneous population as previously considered. Tumor-derived cytokines induce the appearance in the blood of immature neutrophils with immunosuppressive activities (33) and neutrophils with an "aged" phenotype that are experienced cells with increased ability to respond to inflammatory stimuli that can have an anti-tumoral role (34). Also, in the tumor tissue, as macrophages, neutrophils are present in different polarized states: $\mathrm{N} 1$ anti-tumoral phenotype induced by IFN- $\beta$ stimulation and N2 pro-tumoral phenotype induced by TGF- $\beta$ and G-CSF stimulation (35). In addition, several preclinical data have indicated that neutrophils can be activated to kill tumor cells. Neutrophils mediate the therapeutic effect of monoclonal antibodies such as rituximab and trastuzumab in breast cancer $(36)$ and lymphoma $(37,38)$ through antibody-dependent cellular cytotoxicity (ADCC). Neutrophils are the effector cells in the regression of Fas ligand (FasL)-expressing tumors through the expression of Fas (39). 
They also mediate the anti-tumor activity of the PKC modulator ingenol-3-angelate (PEP005) in skin tumors. PEP005 induces the recruitment and the activation of neutrophils that kill the tumor cells by ADCC (40). Neutrophils are again the effector cells in Bacillus Calmette-Guérin-based bladder tumor immunotherapy inducing death of cancer cells by release of TRAIL (41). Interestingly, reactive oxygen species production by neutrophils mediates the immune-mediated abscopal effect of radiotherapy that is tumor regression outside the radiation field (42). Finally, neutrophils are mediating the anti-tumoral activity of the tyrosine kinase inhibitor Cabozantinib in prostate cancer models. Cabozantinib induces the production of neutrophil chemotactic factors such as CXCL12 and HMGB1 that promote a strong infiltration and activation of neutrophils that kill tumor cells (43).

\section{NEUTROPHILS AND GLIOMA}

As for other cancer patients, most of the glioma patients have a strong neutrophilia (44) due to overproduction by tumor cells of G-CSF, the growth factor for neutrophils $(45,46)$. G-CSF diverts BM hematopoiesis away from the lymphocyte lineage toward the granulocyte one and for this reason patients have a high NLR. NLR higher than 4 has been associated with poor prognosis when measured before treatments $(47,48)$, after the second surgery (49), and after temozolomide chemotherapy and radiotherapy (50). NLR less than 4 is associated to better prognosis but only in GBM expressing the wild-type gene IDH1, one of the genes more frequently mutated in malignant gliomas (51). Activation of neutrophils, measured by increased surface expression of $\mathrm{CD} 11 \mathrm{~b}$, is an early predictor of tumor progression in GBM patients (52). Circulating neutrophils promote tumor growth inducing immunosuppression by production of arginase I (53). Interestingly, baseline neutrophil count in blood predicts bevacizumab efficacy in GBM patients. However, neutrophils are not involved in the anti-tumor response but are, on the contrary, the target of the anti-VEGFA therapy being the main producers of this proangiogenic cytokine (54).

Neutrophils are also present within the tumor lesions (55), and there is a correlation between NLR and the degree of neutrophil infiltration (56). The number of infiltrating neutrophils is correlated with glioma grade and with acquired resistance to anti-VEGF therapy in GBM $(55,57)$. Clinical data suggest that neutrophils have a negative prognostic value in GBM patients, but the mechanism of their recruitment and their role in tumor growth are ill defined. In addition, preclinical studies have an important limitation due to differences in relative abundance of

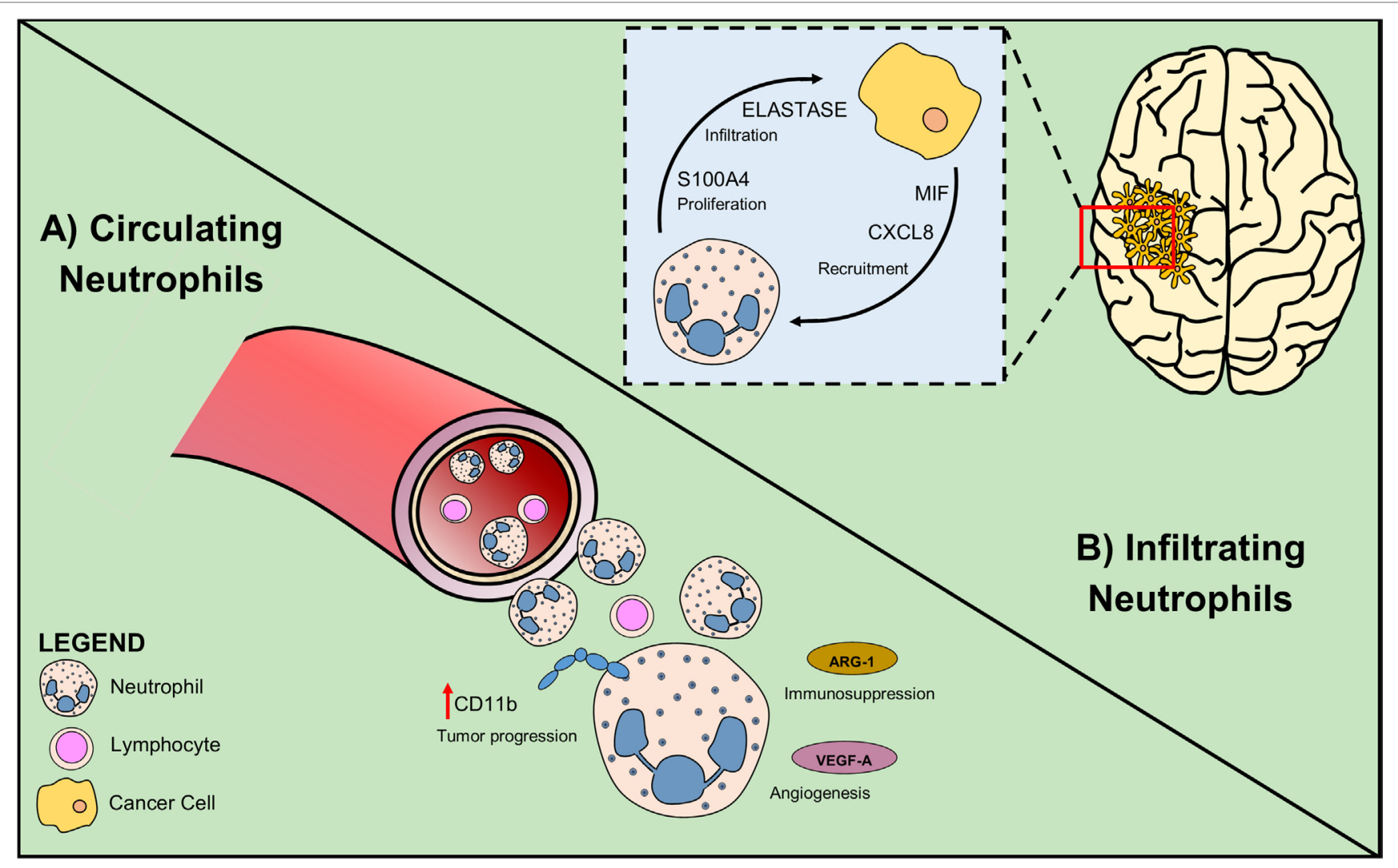

FIGURE 2 | Circulating and infiltrating neutrophils sustain glioma growth. (A) In glioma patients, high NLR is associated with poor prognosis. Neutrophil activation measured as increased CD11b level, is an early sign of tumor progression. Secretion of Arginase-1 by neutrophil promotes immunosuppression and VEGF-A production induces angiogenesis. (B) Glioma cells attract neutrophils by producing the chemoattractants CXCL8 and MIF. Tumor-associated neutrophils exert their pro-tumoral role expressing elastase that sustains glioma infiltration and S100A4 that increases tumor proliferation rate. 
neutrophils in the circulation between human and mice (50-70 and $10-25 \%$, respectively).

Neutrophils are recruited at tumor site by CXCL8 produced by FasL triggering on glioma cells $(58,59)$. Immunosuppressive neutrophils are also recruited by the chemotactic agent MIF produced by glioma cancer stem cells (60). At the tumor site, neutrophils secrete elastase that can aid glioma infiltration (61) and can directly induce the proliferation of GBM-initiating cells by producing S100A4 (57). Neutrophils are also contributing to the resistance to anti-angiogenic therapy (62). Consistently with their pro-tumoral role, depletion of neutrophils using the monoclonal antibody against Ly6G prolonged the survival of mice with developing gliomas (63). Moreover, mutant IDH1 glioma tumors that have a longer survival compared to wild-type IDH1 have a reduced neutrophil infiltration (64).

\section{CONCLUSION AND FUTURE PERSPECTIVES}

To date, most studies performed on glioma patients suggest that neutrophilia and neutrophil infiltration at tumor site have a negative prognostic role in the survival and response to anti-angiogenic therapies (Figure 2). Therefore, most of the approaches are targeted at inhibiting neutrophil migration to the tumor site. Because in other tumors, neutrophils can directly exert an important

\section{REFERENCES}

1. Melin BS, Barnholtz-Sloan JS, Wrensch MR, Johansen C, Il'yasova D, Kinnersley B, et al. Genome-wide association study of glioma subtypes identifies specific differences in genetic susceptibility to glioblastoma and non-glioblastoma tumors. Nat Genet (2017) 49:789-94. doi:10.1038/ ng. 3823

2. Stupp R, Hegi ME, Mason WP, Van Den Bent MJ, Taphoorn MJ, Janzer RC, et al. Effects of radiotherapy with concomitant and adjuvant temozolomide versus radiotherapy alone on survival in glioblastoma in a randomised phase III study: 5-year analysis of the EORTC-NCIC trial. Lancet Oncol (2009) 10:459-66. doi:10.1016/S1470-2045(09)70025-7

3. Wang $\mathrm{H}, \mathrm{Xu} \mathrm{T}$, Jiang $\mathrm{Y}, \mathrm{Xu} \mathrm{H}$, Yan Y, Fu D, et al. The challenges and the promise of molecular targeted therapy in malignant gliomas. Neoplasia (2015) 17:239-55. doi:10.1016/j.neo.2015.02.002

4. Gilbert MR, Dignam JJ, Armstrong TS, Wefel JS, Blumenthal DT, Vogelbaum MA, et al. A randomized trial of bevacizumab for newly diagnosed glioblastoma. N Engl J Med (2014) 370:699-708. doi:10.1056/NEJMoa1308573

5. Ampie L, Woolf EC, Dardis C. Immunotherapeutic advancements for glioblastoma. Front Oncol (2015) 5:12. doi:10.3389/fonc.2015.00012

6. Reardon DA, Freeman G, Wu C, Chiocca EA, Wucherpfennig KW, Wen PY, et al. Immunotherapy advances for glioblastoma. Neuro Oncol (2014) 16:1441-58. doi:10.1093/neuonc/nou212

7. Engelhardt B, Vajkoczy P, Weller RO. The movers and shapers in immune privilege of the CNS. Nat Immunol (2017) 18:123-31. doi:10.1038/ni.3666

8. Louveau A, Smirnov I, Keyes TJ, Eccles JD, Rouhani SJ, Peske JD, et al. Structural and functional features of central nervous system lymphatic vessels. Nature (2015) 523:337-41. doi:10.1038/nature14432

9. Prinz M, Priller J. The role of peripheral immune cells in the CNS in steady state and disease. Nat Neurosci (2017) 20:136-44. doi:10.1038/nn.4475

10. Beena J, Hunter C, Harris TH. Immune cell trafficking in the central nervous system. In: Peterson MTP, editor. Neuroinflammation and Neurodegeneration. New York: Springer (2014). p. 29-45.

11. Harrison-Brown M, Liu GJ, Banati R. Checkpoints to the brain: directing myeloid cell migration to the central nervous system. Int J Mol Sci (2016) 17. doi:10.3390/ijms17122030 antineoplastic activity and improve the efficiency of current therapies, such as anti-cancer antibodies and checkpoint inhibitors, it will be important to uncover this possibility also in glioma.

Consideration should be given to the possibility to improve monoclonal antibody therapies by combination with treatments that promote the differentiation of neutrophils into an anti-tumor phenotype with higher ADCC activity. Interestingly, two treatments, type I IFNs (65) and TGF- $\beta$ targeting (56), that can promote neutrophil differentiation toward an anti-tumoral phenotype are inducing glioma regression. Current therapies may also be combined with treatments that promote selective recruitment of anti-tumoral neutrophils at the tumor site (66). Finally, the ability of neutrophils to migrate in the inflamed brain can be exploited to deliver anti-cancer drugs in gliomas after surgery, reducing regrowth of tumors (67).

\section{AUTHOR CONTRIBUTIONS}

All the authors have contributed to this review by writing and critically evaluating the literature.

\section{FUNDING}

Research activities in the laboratory are supported by the Italian Association for Cancer Research, AIRC IG 2014-15438.

12. Zenaro E, Pietronigro E, Della Bianca V, Piacentino G, Marongiu L, Budui S, et al. Neutrophils promote Alzheimer's disease-like pathology and cognitive decline via LFA-1 integrin. Nat Med (2015) 21:880-6. doi:10.1038/nm.3913

13. He H, Geng T, Chen P, Wang M, Hu J, Kang L, et al. NK cells promote neutrophil recruitment in the brain during sepsis-induced neuroinflammation. Sci Rep (2016) 6:27711. doi:10.1038/srep27711

14. Mantovani A, Allavena P, Sica A, Balkwill F. Cancer-related inflammation. Nature (2008) 454:436-44. doi:10.1038/nature07205

15. Zagzag D, Salnikow K, Chiriboga L, Yee H, Lan L, Ali MA, et al. Downregulation of major histocompatibility complex antigens in invading glioma cells: stealth invasion of the brain. Lab Invest (2005) 85:328-41. doi:10.1038/ labinvest. 3700233

16. Berghoff AS, Kiesel B, Widhalm G, Rajky O, Ricken G, Wohrer A, et al. Programmed death ligand 1 expression and tumor-infiltrating lymphocytes in glioblastoma. Neuro Oncol (2015) 17:1064-75. doi:10.1093/neuonc/nou307

17. Nduom EK, Wei J, Yaghi NK, Huang N, Kong LY, Gabrusiewicz K, et al. PDL1 expression and prognostic impact in glioblastoma. Neuro Oncol (2016) 18:195-205. doi:10.1093/neuonc/nov172

18. Zeng J, See AP, Phallen J, Jackson CM, Belcaid Z, Ruzevick J, et al. Anti-PD-1 blockade and stereotactic radiation produce long-term survival in mice with intracranial gliomas. Int J Radiat Oncol Biol Phys (2013) 86:343-9. doi:10.1016/j.ijrobp.2012.12.025

19. Reardon DA, Gokhale PC, Klein SR, Ligon KL, Rodig SJ, Ramkissoon SH, et al. Glioblastoma eradication following immune checkpoint blockade in an orthotopic, immunocompetent model. Cancer Immunol Res (2016) 4:124-35. doi:10.1158/2326-6066.CIR-15-0151

20. Fecci PE, Ochiai H, Mitchell DA, Grossi PM, Sweeney AE, Archer GE, et al. Systemic CTLA-4 blockade ameliorates glioma-induced changes to the CD4+ $\mathrm{T}$ cell compartment without affecting regulatory T-cell function. Clin Cancer Res (2007) 13:2158-67. doi:10.1158/1078-0432.CCR-06-2070

21. Vom Berg J, Vrohlings M, Haller S, Haimovici A, Kulig P, Sledzinska A, et al. Intratumoral IL-12 combined with CTLA-4 blockade elicits T cellmediated glioma rejection. J Exp Med (2013) 210:2803-11. doi:10.1084/ jem.20130678

22. Wainwright DA, Chang AL, Dey M, Balyasnikova IV, Kim CK, Tobias A, et al. Durable therapeutic efficacy utilizing combinatorial blockade against 
IDO, CTLA-4, and PD-L1 in mice with brain tumors. Clin Cancer Res (2014) 20:5290-301. doi:10.1158/1078-0432.CCR-14-0514

23. Reardon DA, Wen PY, Wucherpfennig KW, Sampson JH. Immunomodulation for glioblastoma. Curr Opin Neurol (2017) 30:361-9. doi:10.1097/WCO. 0000000000000451

24. Pyonteck SM, Akkari L, Schuhmacher AJ, Bowman RL, Sevenich L, Quail DF, et al. CSF-1R inhibition alters macrophage polarization and blocks glioma progression. Nat Med (2013) 19:1264-72. doi:10.1038/nm.3337

25. Quail DF, Joyce JA. The microenvironmental landscape of brain tumors. Cancer Cell (2017) 31:326-41. doi:10.1016/j.ccell.2017.02.009

26. Glass R, Synowitz M. CNS macrophages and peripheral myeloid cells in brain tumours. Acta Neuropathol (2014) 128:347-62. doi:10.1007/s00401014-1274-2

27. Mantovani A, Cassatella MA, Costantini C, Jaillon S. Neutrophils in the activation and regulation of innate and adaptive immunity. Nat Rev Immunol (2011) 11:519-31. doi:10.1038/nri3024

28. Shen M, Hu P, Donskov F, Wang G, Liu Q, Du J. Tumor-associated neutrophils as a new prognostic factor in cancer: a systematic review and meta-analysis. PLoS One (2014) 9:e98259. doi:10.1371/journal.pone.0098259

29. Templeton AJ, Mcnamara MG, Seruga B, Vera-Badillo FE, Aneja P, Ocana A, et al. Prognostic role of neutrophil-to-lymphocyte ratio in solid tumors: a systematic review and meta-analysis. J Natl Cancer Inst (2014) 106:dju124. doi:10.1093/jnci/dju124

30. Caruso RA, Bellocco R, Pagano M, Bertoli G, Rigoli L, Inferrera C. Prognostic value of intratumoral neutrophils in advanced gastric carcinoma in a highrisk area in northern Italy. Mod Pathol (2002) 15:831-7. doi:10.1097/01.MP. 0000020391.98998.6B

31. Sconocchia G, Zlobec I, Lugli A, Calabrese D, Iezzi G, Karamitopoulou E, et al. Tumor infiltration by FcgammaRIII (CD16)+ myeloid cells is associated with improved survival in patients with colorectal carcinoma. Int J Cancer (2011) 128:2663-72. doi:10.1002/ijc.25609

32. Eruslanov EB, Bhojnagarwala PS, Quatromoni JG, Stephen TL, Ranganathan A, Deshpande $\mathrm{C}$, et al. Tumor-associated neutrophils stimulate $\mathrm{T}$ cell responses in early-stage human lung cancer. J Clin Invest (2014) 124:5466-80. doi:10.1172/ JCI77053

33. Zhang D, Chen G, Manwani D, Mortha A, Xu C, Faith JJ, et al. Neutrophil ageing is regulated by the microbiome. Nature (2015) 525:528-32. doi:10.1038/ nature 15367

34. Adrover JM, Nicolas-Avila JA, Hidalgo A. Aging: a temporal dimension for neutrophils. Trends Immunol (2016) 37:334-45. doi:10.1016/j.it.2016.03.005

35. Fridlender ZG, Sun J, Kim S, Kapoor V, Cheng G, Ling L, et al. Polarization of tumor-associated neutrophil phenotype by TGF-beta: "N1" versus "N2" TAN. Cancer Cell (2009) 16:183-94. doi:10.1016/j.ccr.2009.06.017

36. Stockmeyer B, Beyer T, Neuhuber W, Repp R, Kalden JR, Valerius T, et al. Polymorphonuclear granulocytes induce antibody-dependent apoptosis in human breast cancer cells. J Immunol (2003) 171:5124-9. doi:10.4049/ jimmunol.171.10.5124

37. Albanesi M, Mancardi DA, Jonsson F, Iannascoli B, Fiette L, Di Santo JP, et al. Neutrophils mediate antibody-induced antitumor effects in mice. Blood (2013) 122:3160-4. doi:10.1182/blood-2013-04-497446

38. Eruslanov EB, Singhal S, Albelda SM. Mouse versus human neutrophils in cancer: a major knowledge gap. Trends Cancer (2017) 3:149-60. doi:10.1016/j. trecan.2016.12.006

39. Seino K, Kayagaki N, Okumura K, Yagita H. Antitumor effect of locally produced CD95 ligand. Nat Med (1997) 3:165-70. doi:10.1038/nm0297-165

40. Challacombe JM, Suhrbier A, Parsons PG, Jones B, Hampson P, Kavanagh D, et al. Neutrophils are a key component of the antitumor efficacy of topical chemotherapy with ingenol-3-angelate. J Immunol (2006) 177:8123-32. doi:10.4049/jimmunol.177.11.8123

41. Suttmann H, Riemensberger J, Bentien G, Schmaltz D, Stockle M, Jocham D, et al. Neutrophil granulocytes are required for effective Bacillus CalmetteGuerin immunotherapy of bladder cancer and orchestrate local immune responses. Cancer Res (2006) 66:8250-7. doi:10.1158/0008-5472.CAN06-1416

42. Takeshima T, Pop LM, Laine A, Iyengar P, Vitetta ES, Hannan R. Key role for neutrophils in radiation-induced antitumor immune responses: potentiation with G-CSF. Proc Natl Acad Sci U S A (2016) 113:11300-5. doi:10.1073/ pnas. 1613187113
43. Patnaik A, Swanson KD, Csizmadia E, Solanki A, Landon-Brace N, Gehring MP, et al. Cabozantinib eradicates advanced murine prostate cancer by activating anti-tumor innate immunity. Cancer Discov (2017) 7:750-65. doi:10.1158/2159-8290.CD-16-0778

44. Gabrusiewicz K, Rodriguez B, Wei J, Hashimoto Y, Healy LM, Maiti SN, et al. Glioblastoma-infiltrated innate immune cells resemble M0 macrophage phenotype. JCI Insight (2016) 1. doi:10.1172/jci.insight.85841

45. Nitta T, Sato K, Allegretta M, Brocke S, Lim M, Mitchell DJ, et al. Expression of granulocyte colony stimulating factor and granulocyte-macrophage colony stimulating factor genes in human astrocytoma cell lines and in glioma specimens. Brain Res (1992) 571:19-25. doi:10.1016/0006-8993(92)90505-4

46. Albulescu R, Codrici E, Popescu ID, Mihai S, Necula LG, Petrescu D, et al. Cytokine patterns in brain tumour progression. Mediators Inflamm (2013) 2013:979748. doi:10.1155/2013/979748

47. Bambury RM, Teo MY, Power DG, Yusuf A, Murray S, Battley JE, et al. The association of pre-treatment neutrophil to lymphocyte ratio with overall survival in patients with glioblastoma multiforme. J Neurooncol (2013) 114:149-54. doi:10.1007/s11060-013-1164-9

48. Wiencke JK, Koestler DC, Salas LA, Wiemels JL, Roy RP, Hansen HM, et al. Immunomethylomic approach to explore the blood neutrophil lymphocyte ratio (NLR) in glioma survival. Clin Epigenetics (2017) 9:10. doi:10.1186/ s13148-017-0316-8

49. McNamara MG, Lwin Z, Jiang H, Templeton AJ, Zadeh G, Bernstein M, et al. Factors impacting survival following second surgery in patients with glioblastoma in the temozolomide treatment era, incorporating neutrophil/lymphocyte ratio and time to first progression. J Neurooncol (2014) 117:147-52. doi:10.1007/s11060-014-1366-9

50. Mason M, Maurice C, Mcnamara MG, Tieu MT, Lwin Z, Millar BA, et al. Neutrophil-lymphocyte ratio dynamics during concurrent chemoradiotherapy for glioblastoma is an independent predictor for overall survival. J Neurooncol (2017) 132:463-71. doi:10.1007/s11060-017-2395-y

51. Wang PF, Song HW, Cai HQ, Kong LW, Yao K, Jiang T, et al. Preoperative inflammation markers and IDH mutation status predict glioblastoma patient survival. Oncotarget (2017) 8:50117-23. doi:10.18632/oncotarget.15235

52. Rahbar A, Cederarv M, Wolmer-Solberg N, Tammik C, Stragliotto G, Peredo I, et al. Enhanced neutrophil activity is associated with shorter time to tumor progression in glioblastoma patients. Oncoimmunology (2016) 5:e1075693. doi:10.1080/2162402X.2015.1075693

53. Sippel TR, White J, Nag K, Tsvankin V, Klaassen M, Kleinschmidt-Demasters BK, et al. Neutrophil degranulation and immunosuppression in patients with GBM: restoration of cellular immune function by targeting arginase I. Clin Cancer Res (2011) 17:6992-7002. doi:10.1158/1078-0432.CCR-11-1107

54. Bertaut A, Truntzer C, Madkouri R, Kaderbhai CG, Derangere V, Vincent J, et al. Blood baseline neutrophil count predicts bevacizumab efficacy in glioblastoma. Oncotarget (2016) 7:70948-58. doi:10.18632/oncotarget.10898

55. Fossati G, Ricevuti G, Edwards SW, Walker C, Dalton A, Rossi ML. Neutrophil infiltration into human gliomas. Acta Neuropathol (1999) 98:349-54. doi:10.1007/s004010051093

56. Han S, Liu Y, Li Q, Li Z, Hou H, Wu A. Pre-treatment neutrophil-to-lymphocyte ratio is associated with neutrophil and T-cell infiltration and predicts clinical outcome in patients with glioblastoma. BMC Cancer (2015) 15:617. doi:10.1186/s12885-015-1629-7

57. Liang J, Piao Y, Holmes L, Fuller GN, Henry V, Tiao N, et al. Neutrophils promote the malignant glioma phenotype through S100A4. Clin Cancer Res (2014) 20:187-98. doi:10.1158/1078-0432.CCR-13-1279

58. Chio CC, Wang YS, Chen YL, Lin SJ, Yang BC. Down-regulation of Fas-L in glioma cells by ribozyme reduces cell apoptosis, tumour-infiltrating cells, and liver damage but accelerates tumour formation in nude mice. Br J Cancer (2001) 85:1185-92. doi:10.1054/bjoc.2001.2055

59. Hor WS, Huang WL, Lin YS, Yang BC. Cross-talk between tumor cells and neutrophils through the Fas (APO-1, CD95)/FasL system: human glioma cells enhance cell viability and stimulate cytokine production in neutrophils. J Leukoc Biol (2003) 73:363-8. doi:10.1189/jlb.0702375

60. Otvos B, Silver DJ, Mulkearns-Hubert EE, Alvarado AG, Turaga SM, Sorensen MD, et al. Cancer stem cell-secreted macrophage migration inhibitory factor stimulates myeloid derived suppressor cell function and facilitates glioblastoma immune evasion. Stem Cells (2016) 34:2026-39. doi:10.1002/stem.2393 
61. Iwatsuki K, Kumara E, Yoshimine T, Nakagawa H, Sato M, Hayakawa T. Elastase expression by infiltrating neutrophils in gliomas. Neurol Res (2000) 22:465-8. doi:10.1080/01616412.2000.11740701

62. Achyut BR, Shankar A, Iskander AS, Ara R, Angara K, Zeng P, et al. Bone marrow derived myeloid cells orchestrate antiangiogenic resistance in glioblastoma through coordinated molecular networks. Cancer Lett (2015) 369:416-26. doi:10.1016/j.canlet.2015.09.004

63. Fujita M, Scheurer ME, Decker SA, Mcdonald HA, Kohanbash G, Kastenhuber ER, et al. Role of type 1 IFNs in antiglioma immunosurveillance - using mouse studies to guide examination of novel prognostic markers in humans. Clin Cancer Res (2010) 16:3409-19. doi:10.1158/1078-0432.CCR-10-0644

64. Amankulor NM, Kim Y, Arora S, Kargl J, Szulzewsky F, Hanke M, et al. Mutant IDH1 regulates the tumor-associated immune system in gliomas. Genes Dev (2017) 31:774-86. doi:10.1101/gad.294991.116

65. Galani V, Papadatos SS, Alexiou G, Galani A, Kyritsis AP. In vitro and in vivo preclinical effects of type I IFNs on gliomas. J Interferon Cytokine Res (2017) 37:139-46. doi:10.1089/jir.2016.0094
66. Bonavita O, Massara M, Bonecchi R. Chemokine regulation of neutrophil function in tumors. Cytokine Growth Factor Rev (2016) 30:81-6. doi:10.1016/j. cytogfr.2016.03.012

67. Xue J, Zhao Z, Zhang L, Xue L, Shen S, Wen Y, et al. Neutrophil-mediated anticancer drug delivery for suppression of postoperative malignant glioma recurrence. Nat Nanotechnol (2017) 12:692-700. doi:10.1038/nnano.2017.54

Conflict of Interest Statement: The authors declare that the research was conducted in the absence of any commercial or financial relationships that could be construed as a potential conflict of interest.

Copyright (C) 2017 Massara, Persico, Bonavita, Mollica Poeta, Locati, Simonelli and Bonecchi. This is an open-access article distributed under the terms of the Creative Commons Attribution License (CC BY). The use, distribution or reproduction in other forums is permitted, provided the original author(s) or licensor are credited and that the original publication in this journal is cited, in accordance with accepted academic practice. No use, distribution or reproduction is permitted which does not comply with these terms. 